\title{
Un dispositif pour promouvoir et soutenir l'authenticité des interactions en classe de langue seconde
}

\author{
Usha Viswanathan \\ Marie-Elaine Lebel \\ Alena Barysevich \\ Université York \\ Canada
}

\section{Introduction}

Depuis l'approche communicative, on invoque le concept d'authenticité pour faire entrer la vie réelle dans la classe de langue seconde, avec comme prémisse que l'enseignement, pour être efficace, doit préparer avant tout l'apprenant à utiliser la langue à l'extérieur de la salle de classe avec des locuteurs natifs. La référence à des éléments externes à la salle de classe - la vie réelle et le locuteur natif - pour cerner le concept d'authenticité exclut-elle donc la possibilité d'une authenticité inhérente à la salle de classe entre apprenants de langues?

Dans cet article, nous soutenons que l'authenticité ne relève pas uniquement de facteurs extrinsèques, que la salle de classe peut et doit être un lieu d'interactions authentiques. Pour ce faire, nous présentons un dispositif didactique qui promeut et soutient l'authenticité en salle de classe. Dans un premier temps, nous nous intéressons à la notion d'authenticité et présentons des éléments de définition de l'authenticité que nous intégrons dans l'enseignement du français langue seconde (FLS). Nous introduisons ensuite notre dispositif didactique, le groupe de discussion et le suivi des pairs (GDSP) (Lebel et Viswanathan), et présentons deux exemples d'exploitation pour le FLS. En dernière partie, nous démontrons enfin comment la mise en place de ce dispositif instaure une authenticité propre à la salle de classe.

\section{L'authenticité en didactique des langues}

Le concept d'authenticité renvoie dans la littérature en didactique des langues secondes à une multitude de représentations. Nous traiterons ici particulièrement de l'authenticité du texte, de la tâche et de la communication en salle de classe.

\subsection{Le texte authentique}

Les didacticiens s'accordent généralement, à quelques exceptions près ${ }^{1}$, pour définir le texte authentique comme étant «produit dans un but communicatif et non pour l'enseignement de la langue» (Nunan 135; Conseil de l'Europe 11). Le texte authentique a pour objectif de fournir aux apprenants une représentation fidèle de la langue cible, non idéalisée ou simplifiée, et s'oppose au texte fabriqué à des fins pédagogiques. Cependant, si la définition de texte authentique fait l'objet d'un certain consensus, son rôle dans le processus d'apprentissage de la langue cible fait débat en didactique des langues.

D'une part, Breen soutient que le texte authentique, lorsqu'il est utilisé en salle de classe, est sorti de son contexte de production et devient inauthentique. En d'autres mots, les conditions nécessaires pour que le texte soit authentifié par les apprenants et qu'il fasse l'objet d'une utilisation authentique de la langue n'y sont pas réunies. Pour Hutchinson et Waters (159), la question n'est pas tant l'authenticité du texte en lui-même que sa conformité aux objectifs d'apprentissage ${ }^{2}$. Cette position est partagée par Gilmore (98), qui souligne que le véritable enjeu, pour les enseignants, est de présenter des textes qui permettent de former des apprenants capables de communiquer efficacement dans la langue cible, que ces textes soient authentiques ou artificiels. Enfin, bien que l'authenticité soit un concept inhérent à la perspective actionnelle, le Cadre présente l'authenticité des textes plutôt comme une option méthodologique que comme une condition sine qua non de l'enseignement-apprentissage de la L2. Le Cadre énonce, par exemple, que les "textes "authentiques" ou textes fabriqués à des fins pédagogiques, textes de manuels ou textes produits par les apprenants ne sont que des textes parmi d'autres» (Conseil de l'Europe 19) ${ }^{3}$. 
Viswanathan, Usha; Lebel, Marie-Elaine et Alena Barysevich. «Un dispositif pour promouvoir et soutenir l'authenticité des interactions en classe de langue seconde.» Nouvelle Revue Synergies Canada, №11 (2018)

D'autre part, notamment pour les tenants d'une approche par genres de textes, l'authenticité des textes est essentielle dans l'enseignement-apprentissage de la langue (Bawarshi et Reiff; Guimarães-Santos; Hyland; Lousada; Schneuwly et Dolz; entre autres). S'opposant à une conception structuraliste de la langue déconnectée de ses contextes de production, l'approche par genres de textes prend appui sur le texte authentique comme "seules manifestations empiriquement attestables des actions langagières humaines» (Bronckart Activité langagière, textes et discours 12). L'authenticité du texte garantit l'intégration de la dimension discursive et sociolinguistique; l'authenticité du texte incorpore également les normes historiques, sociales et culturelles véhiculées par les genres. En ce sens, un enseignement-apprentissage de la langue basé sur le texte authentique permet un contact et une interaction avec la communauté discursive (Berkenkotter et Huckin). C'est à ce courant que nous souscrivons dans cet article.

\subsection{La tâche authentique}

La définition de l'authenticité de la tâche est une question qui fait aussi débat dans la littérature en didactique. Pour Long et Crookes, une tâche est authentique si elle trouve un écho dans la vie réelle. C'est d'ailleurs à cette définition qu'adhère implicitement le Cadre. Pour Willis, une tâche est authentique si elle met l'accent sur le sens du message et non sur la forme. L'authenticité de la tâche, selon Widdowson, repris par Guariento et Morley, réside plutôt dans l'engagement personnel des apprenants qu'elle suscite. Pour Breen, c'est le processus de négociation entre les acteurs de l'apprentissage qui rend la tâche authentique. Enfin, Ellis (2003, 2009) reprend la distinction de Bachman entre l'authenticité situationnelle, qui a trait au lien avec la vie réelle, et l'authenticité interactionnelle, qui a plutôt lien avec les interactions produites en salle de classe; c'est plutôt sur ce deuxième type d'authenticité qu'Ellis («Task-based language teaching: sorting out the misunderstandings» 227) insiste pour définir une tâche authentique.

Dans cet article, nous soutenons à l'instar d'Ellis $(2003,2009)$ que l'authenticité de la tâche ne se définit pas par rapport à son lien avec la vie réelle, mais plutôt par rapport aux interactions qu'elle suscite. Une tâche authentique est ainsi une tâche qui suscite les mêmes processus d'interactions que ceux suscités dans l'usage naturel de la langue, et pour ce faire, elle se doit de mettre l'accent aussi sur la dimension pragmatique de la communication et d'inclure une lacune d'information qui devra être comblée par les apprenants (Ellis «Taskbased language teaching: sorting out the misunderstandings» 223).

\subsection{La communication authentique en salle de classe}

La salle de classe peut-elle être un lieu d'interactions authentiques? Pour Bange (18) «[d]ans la salle de classe, où le but directeur est l'apprentissage, la communication véritable est tendanciellement réduite, voire éliminée». Toujours selon Bange (20), «le seul enseignement où la communication garde sa structure semble être celui qui s'inspire des modèles canadiens d'immersion qui consistent à enseigner dans la langue et non à enseigner la langue.» Breen (67) s'interroge également sur l'authenticité de la salle de classe, insistant sur le caractère nécessairement artificiel des situations du monde réel qu'on y simule. La salle de classe comme un environnement artificiel d'apprentissage des langues est une idée que l'on retrouve également dans le Cadre, qui indique que "[l]es apprenants s'y engagent dans un "faire-semblant accepté volontairement" pour jouer le jeu de l'utilisation de la langue cible» (Conseil de l'Europe 121). Le Cadre met d'ailleurs davantage l'accent sur la dimension communicative de la salle de classe que sur l'authenticité: «[l]es activités de classe, qu'elles se veuillent "authentiques" ou essentiellement "pédagogiques" sont communicatives dans la mesure où elles exigent des apprenants qu'ils en comprennent, négocient et expriment le sens afin d'atteindre un but communicatif» (Conseil de l'Europe 122).

Si c'est l'idée de communiquer pour la vie réelle avec des locuteurs natifs qui détermine l'authenticité, il est clair que la salle de classe peut difficilement être un lieu d'interactions authentiques. Toutefois, selon Pekarek Doehler (14), «[a]uthenticité [...] ne veut pas dire imitation de situations extrascolaires, mais placement des apprenants en position d'acteurs sociaux face à des besoins, des savoirs, des pertinences, des initiatives et des responsabilités en accord avec la variété des situations communicatives en classe.» L'authenticité n'est donc pas une valeur absolue, mais une valeur relative à une situation d'interaction. Ainsi, il peut exister une authenticité inhérente à la salle de classe se manifestant dans la dimension des interactions, mais cette authenticité ne se définit pas en référence à des éléments externes. Pour nous, elle émerge plutôt de la combinaison de deux éléments, à savoir l'authenticité des textes et l'authenticité de la tâche, qui, mis ensemble, instaurent une authenticité dans la salle de classe. Contrairement à Guariento et Morley qui émettent la possibilité de sacrifier l'authenticité du texte pour garantir l'authenticité des interactions en salle de 
Viswanathan, Usha; Lebel, Marie-Elaine et Alena Barysevich. «Un dispositif pour promouvoir et soutenir l'authenticité des interactions en classe de langue seconde.» Nouvelle Revue Synergies Canada, N¹1 (2018)

classe, nous pensons qu'authenticité du texte et authenticité de la tâche sont nécessairement interdépendantes.

\section{Un dispositif pour la classe de langue seconde}

\subsection{Le GDSP}

Pour soutenir l'authenticité en salle de classe, dans sa dimension interactionnelle, nous avons mis au point un dispositif d'enseignement-apprentissage de la langue, le GDSP (Lebel et Viswanathan). Ce dispositif, élaboré dans une perspective actionnelle (Conseil de l'Europe 2001) et s'inscrivant dans un courant socioconstructiviste de l'apprentissage, se répète de façon hebdomadaire dans les cours de français langue seconde (FLS), à tous les niveaux. Cet outil fait travailler ensemble deux groupes d'étudiants, un groupelocuteur et un groupe-observateur, qui doivent collaborer pour accomplir une tâche intégrant à la fois la compréhension et la production, à l'oral comme à l'écrit.

Le groupe-locuteur est formé de quatre ou cinq étudiants qui ont une tâche de compréhension écrite ou orale, consistant à lire un texte ou à écouter un document audio ou vidéo, suivie d'une tâche de production orale, consistant à discuter du texte écrit ou oral, dans le but, par exemple, de construire une synthèse collective du texte. Le groupe-observateur est aussi formé de quatre ou cinq étudiants qui ont une tâche de compréhension orale, à savoir écouter la discussion du groupe-locuteur, suivie d'une tâche de production écrite, à savoir produire un compte-rendu de la discussion. Les rôles sont ensuite intervertis, c'est-à-dire que le groupelocuteur devient le groupe-observateur et réciproquement.

Au sein du groupe-locuteur, chaque étudiant développe sa propre compréhension du texte, en fonction de sa compétence et de son expérience, et la somme de ces interprétations individuelles mène à une construction collective du sens. Le besoin de négocier un sens commun suscite un grand nombre d'interactions, mais le fait que le groupe-locuteur a une responsabilité envers le groupe-observateur accentue le besoin d'interagir. La lacune d'information vient du fait que la production écrite du groupe-observateur ne peut s'accomplir sans que le groupe-locuteur complète sa tâche de production orale.

Le GDSP est ainsi un dispositif qui constitue une tâche intégrative (Reuter). Cette tâche met en scène une communication exolingue entre locuteurs non natifs (LNN) partageant la même L1 (Porquier). De plus, ce dispositif repose sur l'exploitation des genres de textes pour l'enseignement-apprentissage de la langue, préconisée par l'interactionnisme sociodiscursif (Bronckart, 1996, 1997), par l'École de Genève (Schneuwly et Dolz) et par d'autres chercheurs, comme Lousada et Guimarães-Santos.

\subsection{Deux déclinaisons de tâche intégrative pour deux niveaux de cours de FLS}

Le GDSP peut se décliner de différentes façons selon le contexte et le niveau de cours. Nous souhaitons présenter ici deux exemples concrets. Le premier provient d'un cours de FLS de niveau intermédiaire (B1). Dans cette illustration, la première tâche du groupe-locuteur, en compréhension orale, consiste à visionner à la maison le documentaire Argo, la vraie histoire ${ }^{4}$. Cette tâche de compréhension orale est suivie d'une tâche de production orale: le groupe-locuteur doit construire un résumé collectif du documentaire et en faire une critique. Le groupe-observateur doit quant à lui écouter la discussion du groupe-locuteur et rédiger un compterendu collectif de la discussion.

Le deuxième exemple provient d'un cours de français à vocation universitaire de niveau intermédiaire avancé (B2). Le groupe-locuteur a pour tâche, suite à la lecture de l'essai «Les identités meurtrières» d'Amin Maalouf, d'identifier trois thèmes prédominants de l'essai et chaque étudiant du groupe d'expliquer ces thèmes en faisant des liens avec son domaine d'études ${ }^{5}$. La discussion du groupe-locuteur consiste donc en la présentation des thèmes choisis et des différentes explications de ces thèmes en fonction des disciplines des étudiants. La tâche des étudiants du groupe-observateur consiste à écouter la discussion, à poser, au besoin, des questions de clarification et à rédiger, individuellement, un compte-rendu de discussion. 
Viswanathan, Usha; Lebel, Marie-Elaine et Alena Barysevich. «Un dispositif pour promouvoir et soutenir l'authenticité des interactions en classe de langue seconde.» Nouvelle Revue Synergies Canada, №11 (2018)

\title{
4. La notion d'authenticité à travers le GDSP
}

Le GDSP est un dispositif qui permet d'instaurer une authenticité propre à la salle de classe. Dans ce dispositif, l'authenticité ne repose pas sur des facteurs externes, comme le locuteur natif ou la vie réelle, mais elle émerge des conditions dans lesquelles les interactions prennent place. Ces conditions sont créées par une tâche authentique qui exploite des textes authentiques.

Comme le montrent les sections qui suivent, les tâches à accomplir dans le GDSP déclenchent des interactions authentiques entre apprenants et les motivent à utiliser la langue cible en dépit du fait qu'ils partagent tous la même L1. Ces tâches suscitent également des interactions entre les apprenants et les communautés langagières dont les textes authentiques sont le produit. La combinaison de l'authenticité interactionnelle de la tâche et de l'authenticité des genres de textes établit ainsi le contexte propice pour authentifier la salle de classe et, de surcroit, permet d'instituer un sentiment d'authenticité au sein de la communauté d'apprenants.

\subsection{Interactions authentiques en communication exolingue entre LNN}

Le GDSP est un dispositif qui suscite des interactions authentiques entre apprenants de langues. C'est cette idée que nous permettent de soutenir deux études de cas réalisées à partir d'un corpus de productions, orales et écrites, d'apprenants du FLS. Ces deux études s'intéressent aux stratégies mises en œuvre en interaction. Nous présentons ici sommairement ces deux études, qui sont chacune basées sur une des déclinaisons du GDSP présentées dans la partie 3.2.

Dans la première étude, nous nous sommes intéressées aux stratégies de communication utilisées par les apprenants d'un groupe-locuteur de niveau B1 (Barysevich et al.). Analysées dans une perspective interactionnelle, certaines de ces stratégies, au lieu de témoigner d'un déficit langagier, illustrent plutôt la mise en œuvre du principe de coopération, inhérent à l'usage naturel de la langue. À titre d'exemple, dans l'extrait qui suit ${ }^{6}$, l'étudiante 2 a recours à l'abandon du message comme stratégie compensatoire; on peut cependant observer dans la séquence d'interactions qu'une construction collective du sens se réalise à travers l'échafaudage fourni par l'étudiante 1 et l'étudiant 3 pour amener l'étudiante 2 à formuler ses idées.

\author{
Exemple 1: \\ [Étudiante 2]: [...] qui sont attrapés dans Iran \\ [Étudiante 1]: [se tournant vers l'étudiante 2] Et qu'est-ce qu'il a fait? \\ [Étudiante 2]: Il a fait des passeports, um... \\ [Étudiant 3]: [se tournant vers l'étudiante 2 et allongeant le bras vers elle pour lui donner des \\ suggestions] Et visas? \\ [Étudiante 2]: Et visas \\ [Étudiante 1]: Et quoi l'histoire? Que c'était l'histoire? \\ [Étudiante 2]: II a fait, il a fabriqué
}

Cette étude de cas a ainsi permis de constater que le GDSP encourage l'utilisation d'un large inventaire de stratégies de communication, qu'il incite à une coopération active entre apprenants et qu'il déclenche un grand nombre de demandes d'aide et d'autorégulation des tours de parole. Le concept de lacune d'information inhérent au dispositif suscite un grand nombre d'ajouts d'information et de validation, étant donné la responsabilité du groupe-locuteur envers le groupe-observateur.

Dans la seconde étude, nous nous sommes intéressées à la production d'inférences dans le GDSP (Lebel, Viswanathan et Barysevich, à paraître). Cette étude de cas porte ainsi sur les inférences produites par les étudiants d'un groupe-observateur de niveau B2, qui devaient, à partir de la discussion d'un groupe-locuteur, rédiger un compte-rendu de discussion. En comparant les comptes-rendus avec la transcription de la discussion, nous avons constaté que les observateurs ont fortement recours à l'inférence pour accomplir la tâche de production écrite. Ainsi, la lacune d'information à la base du GDSP susciterait la production de divers types d'inférences, bonnes ou mauvaises, qui font partie de l'usage naturel de la langue ${ }^{7}$. Cette étude permet également de constater que la communication exolingue entre LNN ne constituerait pas un obstacle à la production d'inférences. Enfin, cette étude révèle que le GDSP, en établissant une relation de dépendance entre le groupe-observateur et le groupe-locuteur, crée un besoin pour l'usage de la L2 et répond au défi que mentionnent Jacobs et Kimura (27) d'encourager les participants partageant la même L1 à s'exprimer entre 
Viswanathan, Usha; Lebel, Marie-Elaine et Alena Barysevich. «Un dispositif pour promouvoir et soutenir l'authenticité des interactions en classe de langue seconde.» Nouvelle Revue Synergies Canada, №11 (2018)

eux dans la L2.

En somme, dans les deux cas, l'analyse de données empiriques a montré qu'au sein du GDSP, les apprenants ont recours aux stratégies de négociation du sens, d'échafaudage, d'inférence et de vérification qui sont, selon Ellis («Task-based language teaching» 227) des processus interactionnels présents dans un usage naturel de la langue et définissant l'authenticité interactionnelle. C'est d'abord en ce sens que le GDSP promeut et soutient une authenticité propre à la salle de classe.

\subsection{Genres de textes et interactions avec la communauté langagière}

Notre dispositif, qui se base sur l'exploitation de genres de textes, en réception et en production, promeut et soutient des interactions authentiques entre la communauté d'apprenants et les communautés langagières dont les genres de textes sont le produit. Dans la première déclinaison du GDSP présentée en 3.2, la mise en place du dispositif se base sur le genre de textes documentaire sur un évènement historique à travers la tâche de compréhension orale du groupe-locuteur et le compte-rendu à travers la tâche de production écrite du groupe-observateur. Dans la deuxième déclinaison du GDSP présentée en 3.2, la mise en place du dispositif se base sur le genre de textes essai à travers la tâche de compréhension écrite du groupe-locuteur et également sur le compte-rendu dans la tâche de production écrite du groupe-observateur.

En étant ainsi axé sur les textes authentiques, le GDSP permet aux apprenants de s'approprier les normes historiques et culturelles des genres. En participant, à l'oral et à l'écrit, à l'apprentissage contextualisé des concepts linguistiques dictés par les textes authentiques appartenant à un genre (caractéristiques discursives, textuelles, linguistiques et sociolinguistiques), les apprenants prennent part, collectivement à la manipulation, à l'appropriation et à la reproduction des genres. En outre, comme les genres de textes sont un véhicule par lequel les membres d'une communauté linguistique et culturelle partagent des connaissances, des attitudes, des croyances, des valeurs, des coutumes et des pratiques (Bronckart 1996, 1997; Dolz et Abouzaïd), en agissant sur le texte, non seulement les apprenants s'approprient en interaction le genre, ses structures et ses conventions, mais ils sont aussi mis en relation avec les connaissances, attitudes, croyances, valeurs, coutumes et pratiques d'une communauté. Par exemple, lors de la discussion sur l'essai d'Amin Maalouf «Les identités meurtrières», les apprenants participent à des interactions sur diverses questions sociétales (immigration, religion, mondialisation, cultures, langues, etc.), inhérentes à toute société complexe en mouvement. Le fait que ces apprenants proviennent de différentes communautés culturelles, linguistiques et de différents domaines d'études mène également à une communication interculturelle authentique. C'est ainsi qu'une tâche qui exploite le texte authentique garantit des interactions authentiques avec une communauté langagière en salle de classe.

\subsection{Une communauté d'apprenants authentique et légitime}

Le GDSP promeut et soutient également un sentiment d'authenticité au sein de la communauté d'apprenants. D'une part, ce sentiment d'authenticité émane du fait que dans le GDSP, les apprenants construisent leur propre communauté langagière en poursuivant un but communicatif commun et en établissant des conventions qui structurent leurs productions. D'autre part, ce sentiment d'authenticité découle du fait que le GDSP prend appui sur la communication exolingue entre LNN et la valorise. Cook (195) soutient qu'il faut voir les utilisateurs de L2 comme de véritables locuteurs et non comme des imitations du locuteur natif. Au sein du GDSP, les apprenants sont susceptibles de devenir des locuteurs authentiques et légitimes d'une communauté langagière, sans avoir besoin de la validation de la communauté cible. Comme le mentionnent Kramsch et Sullivan, citées par Cook (188), le concept de locuteur natif impose des normes d'interaction au lieu de permettre à la communauté d'apprenants de créer ses propres normes. Or, nos études sur les interactions dans le GDSP révèlent l'émergence d'une norme communicative d'origine endogène, c'est-à-dire une norme issue de la communauté d'apprenants, et non une norme imposée par des facteurs externes. En ce sens, le GDSP contribue à estomper la distinction entre apprenant et utilisateur de la langue; il permet d'envisager la communication exolingue entre LNN en des termes positifs et non en termes déficitaires.

\section{Conclusion}

Cet article a eu comme objectif de remettre en question la référence à deux éléments externes - le locuteur natif et la vie réelle - comme la condition préalable de l'authenticité en salle de classe de langues secondes. Nous avons suggéré que la salle de classe peut et doit être authentique, et que cette authenticité relève de la 
Viswanathan, Usha; Lebel, Marie-Elaine et Alena Barysevich. «Un dispositif pour promouvoir et soutenir l'authenticité des interactions en classe de langue seconde.» Nouvelle Revue Synergies Canada, N¹1 (2018)

combinaison de deux éléments interdépendants: le texte authentique et la tâche authentique. Véhiculant autant les dimensions linguistiques, discursives, sociolinguistiques que des normes historiques, sociales et culturelles, le texte authentique est un élément clé pour faire le pont entre une communauté d'apprenants et des communautés linguistiques et culturelles. Mais c'est aussi la tâche à effectuer à partir du texte - la tâche intégrant la dimension pragmatique de la communication et une lacune d'information à être comblée par les apprenants de façon collaborative - qui permet de susciter des interactions authentiques.

Pour mettre en place les conditions de l'authenticité en salle de classe, nous avons présenté un dispositif didactique, le GDSP, qui promeut l'authenticité des apprenants de L2, définie par la combinaison de l'authenticité interactionnelle de la tâche et des genres de textes. En soutenant que l'authenticité est un construit social relatif à une situation d'interaction, ce dispositif forme des acteurs sociaux activement impliqués dans la construction collective de leur propre communauté linguistique, sans se limiter aux normes de la communauté de la langue cible. Ce dispositif élaboré à partir de la notion de communauté d'apprenants fonctionne grâce à la coopération, à un usage de la langue contextualisé et à des échanges réciproques entre apprenants, ayant des connaissances, expériences, parcours, cultures et identités multiples. L'adhésion à cette communauté contribue à forger parmi les apprenants de L2 un sentiment d'appartenance identitaire et une légitimité. Pour citer Tatsuki (3): «We create our own sense of authenticity through social interactions, through our use of language». Être membre de la communauté langagière authentique et légitime contribue au renforcement de la motivation pour apprendre une langue seconde et à la responsabilisation et à l'image positive de l'apprenant.

\section{Notes}

${ }^{1}$ Voir entre autres Bacon et Finneman (469) qui définissent le texte authentique comme étant un texte produit par un locuteur natif pour un locuteur natif.

2 "Fitness to the learning purpose."

${ }^{3}$ La position défendue par les auteurs du Cadre sur le rôle des textes authentiques dans l'apprentissage des langues se rapproche de celle de Hutchinson et Waters et de Gilmore, comme on peut le constater à la lecture de la section 6.4.3 du Cadre où il apparait explicitement que le texte authentique est une option méthodologique devant avant tout servir des objectifs d'apprentissage (Conseil de l'Europe 112-113).

${ }^{4}$ Ce documentaire produit par Radio-Canada critique les inexactitudes historique du film Argo, gagnant de l'Oscar du meilleur film de l'année en 2012, et revient sur le rôle du Canada dans l'évasion de six diplomates américains lors du siège de l'ambassade des Etats-Unis à Téhéran, en 1980.

${ }^{5}$ Dans une même classe de FLS, les étudiants proviennent de domaines d'études divers, par exemple la science politique, l'économie, la psychologie, etc.

${ }^{6}$ Cet extrait fait partie d'une séquence d'interactions où les membres du groupe-locuteur tente d'expliquer les circonstances de l'évasion des otages de l'ambassade américaine à Téhéran selon le documentaire Argo, la vraie histoire.

${ }^{7}$ Ces inférences sont soit des inférences de cohérence, nécessaire à la compréhension du discours, ou des inférences d'élaboration, non nécessaires, mais contribuant à l'enrichissement du texte (Fayol; Gaonac'h et Golder). Elles sont soit basées sur le texte, soit sur les connaissances de l'étudiant. Enfin, elles peuvent témoigner d'une bonne ou d'une mauvaise interprétation du contenu ou des intentions, selon leur compatibilité avec le discours original. 
Viswanathan, Usha; Lebel, Marie-Elaine et Alena Barysevich. «Un dispositif pour promouvoir et soutenir l'authenticité des interactions en classe de langue seconde.» Nouvelle Revue Synergies Canada, N¹1 (2018)

\section{Bibliographie}

Bacon, Susan M., et Michael D. Finneman. «A Study of the Attitudes, Motives, and Strategies of University Foreign Language Students and Their Disposition to Authentic Oral and Written Input.» The Modern Language Journal, vol. 74, no. 1, 1990, pp. 459-473. Disponible à dx.doi.org/10.1111/j.1540-

4781.1990.tb05338.x.

Bachman, Lyle F. Fundamental Considerations in Language Testing. Oxford University Press, 1990.

Bange, Pierre. «À propos de la communication et de l'apprentissage de L2 (notamment dans ses formes institutionnelles).» Acquisition et interaction en langue étrangère, 1992.

Barysevich, Alena, et al. «Stratégies de communication et de négociation du sens dans un cours de français à vocation universitaire: étude de cas.» Recherche et pratiques pédagogiques en langue de spécialité, Cahiers de l'APLIUT, vol. XXXV, no. 1, 2016.

Bawarshi, Anis S., et Marie J. Reiff. Genre. An Introduction to History, Theory, Research, and Pedagogy. Parlor Press and The WAC Clearinghouse, 2010.

Berkenkotter, Carol, et Thomas N. Huckin. «Rethinking Genre from a Sociocognitive Perspective.» Written Communication, vol. 10, no. 4, 1993, pp. 475-509.

Breen, Michael P. «Authenticity in the language classroom.» Applied Linguistics, vol. 6, no. 1, 1985, pp. 60-70.

Bronckart, Jean-Paul. «L'acquisition des discours. Le point de vue de l'interactionnisme socio-discursif.»Le français dans le monde, 1996, pp. 55-64.

---. Activité langagière, textes et discours. Pour un interactionisme sociodiscursif. Delachaux et Niestlé, 1997.

Conseil de l'Europe. Cadre européen commun de référence pour les langues: Apprendre, enseigner, évaluer. Les Éditions Didier, 2001.

Cook, Vivian. «Going beyond the native speaker in language teaching.» TESOL Quarterly, vol. 33, no. 2, 1999, pp. 185-209.

Dolz, Joaquim, et Miriam Abouzaïd. «Développer des compétences pour enseigner la production écrite en Suisse romande.» Le Français aujourd'hui, vol. 191, no. 4, 2015, pp. 85-96, Armand Colin. www.revues.armand-colin.com/lettres-langues/francais-aujourdhui/francais-aujourdhui-ndeg-19142015/developper-competences-enseigner-production-ecrite-suisse-romande.

Ellis, Rod. «Task-based language teaching: sorting out the misunderstandings.» International Journal of Applied Linguistics, vol. 19, no. 3, 2009, pp. 221-246.

---. Task-based language learning and teaching. Oxford University Press, 2003.

Fayol, Michel. «Comprendre ce qu'on lit: De l'automatisme au contrôle.» M. Fayol, J.E. Gombert, P. Lecocq, L. Sprenger-Charolles et D. Zagar (eds.), Psychologie cognitive de la lecture. Presses universitaires de France, 1992.

Gaonac'h, Daniel, et Caroline Golder. Lire et comprendre - Psychologie de la lecture. Hachette, 1998.

Gilmore, Alex. «Authentic materials and authenticity in foreign language learning.» Language Teaching, vol. 40, no. 2, 2007, pp. 97-118.

Guariento, William, et John Morley. «Text and Task Authenticity in the EFL Classroom.» ELT Journal, vol. 55, 
Viswanathan, Usha; Lebel, Marie-Elaine et Alena Barysevich. «Un dispositif pour promouvoir et soutenir l'authenticité des interactions en classe de langue seconde.» Nouvelle Revue Synergies Canada, N¹1 (2018)

no. 4, 2001, pp. 347-353.

Guimarães-Santos, Luiza. «Perspective actionnelle et genres textuels: le modèle didactique dans l'enseignement du français langue étrangère.» Synergies Canada, vol. 5, 2012, pp. 1-16. doi: 10.21083/synergies.v0i5.1686.

Hutchinson, Tom, et Alan Waters. English for Specific Purposes: A Learning Centred Approach. Cambridge University Press, 1987.

Hyland, Ken. «Genre Pedagogy: Language, Literacy and L2 Writing Instruction.» Journal of Second Language Writing, vol. 16, no. 3, 2007, pp. 148-164.

Jacobs, George M., et Harumi Kimura. «Encouraging Second Language Use in Cooperative Learning Groups.» Beyond Words, vol. 1, n 1, 2013, pp. 23-36.

Kramsch, Claire, et Patricia Sullivan. «Appropriate pedagogy.» ELT Journal, vol. 50, no. 3, 1996, pp. $199-212$.

Lebel, Marie-Élaine, et Usha Viswanathan. «Une séquence didactique en français langue seconde: le groupe de discussion comme objet et comme dispositif authentique d'enseignement à l'université.» Coedeiro, G.S, et D. Vrydachs (eds.) Les genres dans l'enseignement du français: un objet ou/et un outil didactique? Presses universitaire de Namur \& AIRDF, collection Recherches en didactique du français, vol. 7, 2016, pp. 279-301.

Lebel, Marie-Élaine et al. «Interaction orale et production écrite: inférer pour mieux communiquer.» Cahiers de praxématique, no. 68 , à paraitre.

Long, Michael H., et Graham Crookes. «Three approaches to task-based syllabus design.» TESOL Quarterly, vol. 26, no. 1, 1992, pp. 27-56.

Lousada, Eliane. «Utiliser les genres textuels dans une perspective actionnelle.» Revista de Lenguas Modernas, vol. 1, 2008, pp. 211-218.

Maalouf, Amin. Les identités meurtières. Éditions Grasset \& Fasquelle, 1998.

Nunan, David. The learner-centered curriculum. Cambridge University Press, 1988.

Pekarek Doehler, Simona. «L'interaction en classe de français langue seconde: lieu acquisitionnel - lieu social.» Babyloni, vol. 4, no. 94, 1994, pp.12-16.

Porquier, Rémy. «Communication exolingue et apprentissage des langues.» Acquisition d'une langue étrangère (Actes du Colloque de Neuchâtel, 16-18 septembre 1982), Universités de Paris VIII et de Neuchâtel, 1984, pp. 17-47.

Reuter, Yves. «Statut et usages de la notion de genre en didactique(s).» Le Français aujourd'hui, vol. 159, 2007, pp. 11-18.

Schneuwly, Bernard, et Joaquim Dolz. «Les genres scolaires; des pratiques langagières aux objets d'enseignement.» Repères, vol. 15, 1997, pp. 27-40.

Tatsuki, Donna. Authentic Communication: Proceedings of the 5th Annual JALT Pan-SIG Conference. Tokai University College of Marine Science, 2006, pp. 1-15.

Widdowson, Henry G. Teaching Language as Communication. Oxford University Press, 1978.

Willis, Jane. A framework for task-based learning. Longman, 1996. 